\title{
Réponse analytique à une intoxication par substances volatiles
}

\section{Analytical response following exposure to volatile compounds}

\section{RÉSUMÉ}

Dans notre société, de nombreux produits contiennent des proportions plus ou moins importantes de substances volatiles. On les retrouve par exemple dans les articles domestiques tels que nettoyants, colles, peintures, etc. Les substances volatiles et autres solvants organiques sont aussi largement utilisés dans le domaine industriel et les laboratoires. En milieu médical, leur utilisation s'est développée en anesthésiologie.

Ces substances, lorsqu'elles sont inhalées, produisent des effets sur l'organisme similaires à ceux induits par les dépresseurs du système nerveux central. L'exposition accidentelle, chronique ou intentionnelle (toxicomanie) aux substances volatiles est donc un réel problème de santé publique.

Lors d'une intoxication, les symptômes et signes cliniques observés peuvent constituer des indices facilitant la tâche du toxicologue, mais l'identification formelle du toxique ne pourra être apportée qu'après analyse des échantillons biologiques par une technique appropriée.

Cet article tente de répertorier les outils analytiques pour la mise en évidence d'une intoxication par substances volatiles.

MOTS-CLÉS

Intoxication, volatiles, toxicologie, dosage.

\section{SUMMARY}

Human daily uses products which contain substantial amounts of volatile substances. Common household products which often contain organic solvents include cleaning and polishing fluids, contact adhesives, paint and nail-polish removers. Volatile compounds and organic solvents are also used extensively in industry and laboratories. Some of them are used as anesthetics in hospital.

Many volatile substances, if inhaled in sufficient quantity, produce effects similar to those of central nervous system depressants such as ethanol or barbiturates. Acute or chronic exposure to solvent vapour as a result of industrial, medical and domestic use of these substances is thus a common occurrence.

During intoxication with a volatile compound, the clinical signs can help the analyst in the research of the responsive toxic, but the univocal identification of the substance will be done only after analysis of the biological samples by an appropriate procedure.

This article reviews the analytical techniques for the identification of an intoxication by volatile compounds.

\section{KEY-WORDS}

Intoxication, volatile compounds, toxicology, analysis. 


\section{Introduction}

Les substances volatiles appartiennent à une famille chimique de composés ayant la propriété de passer spontanément ou facilement, de leur état initial à l'état de vapeur. Ce groupe renferme essentiellement les solvants (halogénés ou non) et les gaz (azote, dioxyde de carbone, fluorocarbones, etc.), avec des produits chimiques tels que les alcanes, les hydrocarbures, les éthers, les esters, etc.

Leur utilisation a été généralisée dans de nombreux secteurs. En service hospitalier, certaines d'entre-elles sont utilisées comme anesthésiques (protoxyde d'azote, trichlorométhane, halothane, diéthyléther, enflurane, etc.). Dans la vie courante, nombre de produits finis comme les aérosols (produits cosmétiques ou ménagers, bombes lacrymogènes, etc.), les colles ou les peintures en renferment dans des proportions plus ou moins importantes. En milieu industriel, les solvants organiques sont largement utilisés pour dissoudre d'autres composés et notamment les graisses. Enfin, les sites naturels, volcans, sousterrains ou gisements de pétrole, sont à l'origine de l'émission de certains gaz comme l'hydrogène sulfuré.

Leurs propriétés thermodynamiques (volatilité) et pharmacodynamiques (liposolubilité) en font des produits particulièrement dangereux car ils peuvent pénètrer sans difficultés dans l'organisme par les poumons et induire des effets sur le système nerveux central similaires à ceux provoqués par certains dépresseurs (barbituriques ou benzodiazépines). Leurs effets vont de la simple euphorie à des états de convulsions, de dépression respiratoire et de coma pouvant conduire au décès de l'individu exposé. Généralement, les mesures de prévention permettent d'éviter l'exposition du travailleur à une concentration élevée en toxique, responsable de maladies professionnelles et parfois d'accidents du travail. Dans le cadre d'une toxicomanie, l'exposition au risque est délibérée. Les substances toxiques qui donnent naissance à des toxicomanies en milieu industriel sont les solvants (1), mais cette toxicomanie se rencontre très souvent en dehors du milieu professionnel, surtout chez les adolescents $(2,3)$. Parmi les caractéristiques générales de cette toxicomanie, il faut insister sur la grande facilité d'obtention de ces solvants ou de produits en contenant (colle, déodorisant, décapant, etc.) ainsi que sur leur coût très faible, ce qui en fait parfois des drogues très bon marché. L'exposition, accidentelle, chronique ou intentionnelle (toxicomanie), aux substances volatiles est donc un réel problème de santé publique.

Lors d'une intoxication, les symptômes et signes cliniques observés, les lieux (hôpital, laboratoires), les circonstances, l'odeur caractéristique, ou la présence de l'arsenal du toxicomane (sacs, flacons ou aérosols, etc.) peuvent constituer des indices facilitant la tâche du toxicologue, mais l'identification formelle du toxique ne pourra être apportée qu'après analyse des échantillons biologiques par une technique appropriée.

Le choix de la méthode est conditionné par la rapidité d'obtention du résultat et du degré de spécificité désiré. Les méthodes d'identification et de dosage des substances volatiles dans les fluides biologiques se subdivisent en deux grands groupes, les tests colorimétriques et les techniques chromatographiques. Cet article résume ces différents outils analytiques pour la recherche et la mise en évidence d'une intoxication par les substances volatiles.

\section{Prélèvement et conserva- tion des échantillons}

En cas d'investigations toxicologiques lors d'une intoxication par substances volatiles, certaines précautions concernant le mode de prélèvement et la conservation des échantillons doivent être prises et plus particulièrement pour des substances très volatiles comme le propane, le butane et autres propulseurs d'aérosols.

Certaines substances volatiles sont relativement stables dans le sang si certaines précautions sont prises. Le flacon de stockage doit être en verre et sertis avec une capsule métallique montée avec un septum. Les pertes peuvent être plus importantes si les flacons utilisés sont en polypropylène. Ceci a été démontré par exemple pour le trichloroéthylène où des concentrations sanguines létales peuvent disparaitrent 30 jours après stockage de l'échantillon dans des tubes en plastique (4). Il est préférable que les flacons soient remplis au maximum et ouvert uniquement au moment de l'analyse et lorsqu'ils sont froids (5). Un anticoagulant (héparine, lithium ou EDTA) devrait être ajouté au sang pour éviter la formation de caillots et le prélèvement doit être stocké entre -20 et $4^{\circ} \mathrm{C}(6)$. Pour les esters comme les acétates d'éthyle ou de méthyle, il est conseillé d'additionner du fluorure de sodium $(1 \% \mathrm{w} / \mathrm{v})$ pour réduire l'activité estérasique (6).

Puisque de nombreux solvants et gaz sont nécessaires à l'activité des laboratoires d'analyse, des précautions toutes particulières doivent être prises pour éviter tout risque d'interférence avec les analyses. Bien évidement, les produits purs doivent être soigneusement conditionnés et stockés à part des prélèvements biologiques, pour éviter toute contamination.

Lors de décès imputables à des substances volatiles, l'analyse de tissus riches en lipides (l'encéphale par 
exemple) peut confirmer les très faibles concentrations sanguines car ils sont des sites privilégiés de fixation. Les autres tissus peuvent aussi être analysés; ils doivent être conservés comme le sang. L'analyse urinaire quant à elle permettra de déterminer la présence de métabolites $(7,8,9)$.

\section{Tests colorimétriques}

Les tests colorimétriques pour la détection des substances volatiles sont généralement simples à mettre en oeuvre, nécessitent peu d'échantillon et de réactifs, et apportent une réponse quasi-instantanée. En présence de réactifs chimiques appropriés, les substances cibles donnent des couleurs relativement distinctes. Dans certains cas, la coloration obtenue avec un réactif bien défini peut être spécifique d'un toxique, mais en général, la réaction colorée est spécifique de différents composés d'une même famille, voire de familles différentes. L'intensité de la coloration est certe fonction de la concentration en toxique mais les résultats restent bien souvent qualitatifs puisque des variations de teinte apparaissent suivant les conditions dans lesquelles a été effectué le test. Quelque soit le test colorimétrique mis en oeuvre, l'interprétation du résultat doit être faite par comparaison à des échantillons de référence (témoins positifs et négatifs) testés en parallèle, dans les mêmes conditions. Dans certains cas, il est possible d'appliquer une étape chromatographique sur couche mince avant la coloration pour palier aux risques d'interférence lorsque plusieurs toxiques sont présents.

Les tests colorimétriques les plus courant pour la recherche de substances volatiles sont les suivants :

- le test Fujiwara pour la détection des composés trichlorés,

- le test de dilution pour la recherche du monoxyde de carbone $(\mathrm{CO})$, et

- la désorption en cellules de Conway pour la recherche des cyanures.

1) Le test Fujiwara (10) est un test colorimétrique qui détecte la présence de composés trichlorés. Pour leur recherche au niveau urinaire, il suffit d'ajouter un volume de soude et de pyridine à $1 \mathrm{ml}$ d'urines et de chauffer à $100^{\circ} \mathrm{C}$ pendant 2 minutes. L'apparition d'une coloration rouge-violet au niveau de la pyridine sera indicateur d'une exposition à un toxique trichloré. Deux échantillons de référence, l'un positif, l'autre négatif, devront être traités en même temps pour valider les résultats. La nature du toxique trichloré ne peut pas être déterminée ainsi, mais la positivité du test oriente rapidement l'analyste. Dans certains cas, une intoxication par tétrachlorure de carbone ne pourra être décelée par ce test car la métabolisation du toxique en composés trichlorés est très faible.

2) L'intoxication par le monoxyde de carbone (CO) constitue encore une des principales causes de décès d'origine toxique en France. Les accidents surviennent générallement en hiver lors de l'utilisation d'un chauffage défectueux. L'intoxication au CO peut être mise en évidence rapidement en diluant un volume de sang avec 19 volumes d'ammoniac $0,01 \mathrm{M}$ afin de voir apparaître une teinte rosée évocatrice d'une hémoglobine partiellement saturée en CO.

La carboxyhémoglobinémie peut être déterminée (11): - soit directement par mesure des maximums d'absorption spécifiques pour l'oxyhémoglobine ( $\lambda \max 576,6$ et $541,5 \mathrm{~nm})$ et la carboxyhémoglobine ( $\lambda \max 572,7$ et $539,9 \mathrm{~nm}$ ),

- soit par mesure du CO libéré (après dénaturation acide) grâce à ses propriétés réductrices (coloration rouge par la méthode de Le Moan).

3) La majorité des intoxications par les cyanures surviennent au cours de la manipulation de produits cyanogènes en milieu industriel et lors d'incendies. Parfois, l'intoxication est observée après tentative d'autolyse. La recherche des cyanures peut se faire par réaction de désorption en cellules de Conway. Dans le compartiment inférieur de la cellule sont ajoutés $0,5 \mathrm{ml}$ d'acide sulfurique $3 \mathrm{M}, 4$ grenailles de zinc et $0,5 \mathrm{ml}$ de l'échantillon. Après fermeture hermétique de la cellule et pendant l'étape d'agitation, les cyanures diffusent et sont piégés par la solution de soude $0,1 \mathrm{M}$ placée préalablement dans la cupule supérieure. Un volume de soude est alors prélevé $(0,1 \mathrm{ml})$ auquel est ajouté $1 \mathrm{ml}$ de tampon $\mathrm{NaH}_{2} \mathrm{PO}_{4} 1 \mathrm{M}$ et $0,5 \mathrm{ml}$ de chloramine $\mathrm{T}$. Après 3 min de contact, un réactif colorant (acide barbiturique) est ajouté et 10 minutes plus tard, il est possible d'observer une coloration rouge. Une gamme étalon permettra de quantifier les cyanures après lecture à $580 \mathrm{~nm}$. Une limite de cette technique est le manque de sensibilité pour la mesure des taux physiologiques.

\section{Méthodes chromatogra- phiques}

Hormis la spectrométrie de masse (SM) directe de l'air expiré (permettant de détecter différents composés quelques heures après une exposition), les techniques de recherche des substances volatiles nécessitent une étape préparative de l'échantillon biologique suivie de la séparation chromatographique des composés injectés. Ces techniques peuvent être essentiellement classées en deux sous-groupes distincts en fonction de 
l'étape préparative utilisée:

- la microextraction sur fibre (SPME ou solid-phase microextraction)

- la préparation par "espace de tête".

\section{La microextraction par fibre}

Une excellente revue de la littérature a été récemment publiée (12).

La microextraction sur phase solide a été introduite pour l'isolement des contaminants de l'air et de l'eau. Cette technique a ensuite été utilisée pour la détection des arômes dans les aliments, des résidus de solvants dans les produits pharmaceutiques, mais aussi pour l'analyse d'échantillons biologiques (sang, urines). Elle est simple à mettre en oeuvre, rapide, peu coûteuse, compatible avec les systèmes d'analyse habituels (chromatographie en phase gazeuse ou liquide, électrophorèse capillaire) et automatisable. Cette technique peut aussi être directement couplée à la spectrométrie de masse. Contrairement aux extractions classiques (liquide-liquide ou solide-liquide), la SPME ne fait pas appel à l'utilisation de solvants toxiques.

Cette technique est basée sur la partition des analytes entre la fibre (phase d'extraction) et l'échantillon. La fibre est un petit tube de silice recouvert d'une phase polymérique et montée dans une seringue ayant la possibilité de rétracter la fibre dans sa partie interne. L'extraction est réalisée par immersion de la fibre directement dans l'échantillon ou dans sa preparation "espace de tête" (voir paragraphe suivant). Ensuite, la fibre est introduite dans l'analyseur. Pour la séparation en phase gazeuse (CPG), la thermodésorption des analytes est réalisée dans l'injecteur du chromatographe. Pour la séparation en phase liquide (HPLC), les analytes piègés dans la fibre sont solubilisés par un solvant approprié et injectés par l'intermédiaire d'une boucle. Les propriétés physiques et chimiques des phases commercialisées (polydiméthylsiloxane, polyacrylate, polydiméthylsiloxane-divinylbenzène, etc.), mais aussi l'épaisseur de phase, sont essentielles dans le processus de partition. La durée de vie limitée des fibres (obturation des pores de la phase par les matrices complexes) constitue la limitation majeur de cette technique. Concernant les intoxications par substances volatiles, Brewer et al. (13) rapportent deux cas d'accidents de la route: Dans le premier cas, la préparation "espace de tête" suivie de la microextraction sur fibre a permis d'identifier du dichlorométhane dans les urines de la victime à la concentration de $23 \mathrm{mg} / \mathrm{L}$ (seule une faible quantité de dichlorométhane est retrouvé inchangé dans les urines). Des alcanes, contenus dans les briquets et leurs recharges, ont pu être détectés dans le contenu gastrique de la deuxième victime.
Un cas d'intoxication mortelle suite à une exposition accidentelle au tétrachloroéthylène d'une femme a été décrit par Dehon et al. (14). Le tétrachloroéthylène, le trichloroéthylène et leurs métabolites ont pu être quantifié dans le sang, les urines et les tissus de la victime après extraction par les fibres SPME et analyse par $\mathrm{CPG} / \mathrm{SM}$. Le tableau I résume les taux de tétrachloroéthylène déterminés dans les échantillons biologiques de la victime après son hospitalisation.

Tableau I : Concentrations en tétrachloroéthylène dans les fluides biologiques et divers échantillons tissulaires suite à une intoxication aiguë (14).

\begin{tabular}{|c|c|c|}
\hline Échantillon & Moment du prélèvement & Concentration \\
\hline Sang & $2^{\text {mex }}$ jour & $1319 \mathrm{ng} / \mathrm{ml}$ \\
\hline Sang & $7^{\text {2nx }}$ jour (au décès) & $656 \mathrm{ng} / \mathrm{ml}$ \\
\hline Urines & $2^{\text {ene }}$ jour & $93 \mathrm{ng} / \mathrm{ml}$ \\
\hline Urines & $7^{\text {tane jour (au décès) }}$ & non détecté \\
\hline Foie & post mortem & $1950 \mathrm{ng} / \mathrm{g}$ \\
\hline Myocarde & post mortem & $1678 \mathrm{ng} / \mathrm{g}$ \\
\hline Muscle & post mortem & $751 \mathrm{ng} / \mathrm{g}$ \\
\hline Rein & post mortem & $1195 \mathrm{ng} / \mathrm{g}$ \\
\hline Cortex & post mortem & $1018 \mathrm{ng} / \mathrm{g}$ \\
\hline Bulbe & post mortem & $1855 \mathrm{ng} / \mathrm{g}$ \\
\hline Cervelet & post mortem & $912 \mathrm{ng} / \mathrm{g}$ \\
\hline
\end{tabular}

\section{La préparation "espace de tête" ou head- space (HS)}

La préparation "espace de tête" consiste à faire passer à l'état de vapeur les substances volatilisables pouvant être contenues dans un échantillon. Pour cela, l'échantillon à analyser est introduit dans un flacon en verre hermétiquement scellé et incubé à température élevée pendant un temps déterminé. Afin de favoriser et d'augmenter le passage à l'état de vapeur des composés, les prélèvements biologiques (sang, urines) peuvent être saturés par des sels tels que chlorures de sodium ou de potassium. La préparation "espace de tête" est appliquable aux fluides biologiques habituels (sang, urines), mais aussi aux tissus lorsqu'il s'agit d'intoxications à l'issue fatale. Dans ce cas, le protocole prévoit souvent une étape supplémentaire vissant à digérer le tissu biologique afin de libérer les substances recherchées. Après volatilisation, un aliquot de la phase gazeuse est prélevée et injectée dans l'analyseur. Les étapes préparatives d'incubation et d'injection peuvent être automatisées conduisant les industriels à proposer des couplage on-line HS-CPG-détecteur. Les colonnes chromatographiques classiquement utilisées ont un greffage polaire de type polyéthylène glycol (dosage des solvants), apolaire de type $5 \%$ phényl-95\% méthylsi- 
loxane (dosage du chloralose, des fréons), ou constituent tout simplement un tamis moléculaire (dosage du monoxyde de carbone). La préparation "espace de tête" d'un échantillon suivie d'une analyse par chromatographie en phase gazeuse et détection par un détecteur à capture d'électron (ECD) ou à ionisation de flamme (FID) représente la méthode de choix pour la recherche de substances volatiles dans les diverses matrices biologiques (15). Pour plus de spécificité dans l'identification, le couplage CPG/SM est intéressant, mais l'interprétation devient plus délicate pour des substances volatiles générant des ions de faible masse (6). Dans certains cas, la préparation "espace de tête" peut être couplée directement à la spectrométrie de masse.

Les cas d'intoxications par substances volatiles mis à jour à l'aide de ce type de couplage peuvent concerner le monoxyde de carbone, les cyanures, les solvants (halogènés ou non), les gaz propulseurs (fluorocarbones), les gaz lacrymogènes, les gaz naturels (hydrogène sulfuré), les aphrodisiaques (poppers) ou les anesthésiques. La mise en évidence d'une intoxication peut se faire par la recherche de la substance volatile inchangée dans le sang ou l'air expiré puisque beaucoup d'entre-elles sont éliminées sous leur forme initiale. Certaines sont partiellement éliminées dans l'air expiré et métabolisées dans le foie; les métabolites formés sont alors éliminés dans l'air expiré ou dans les urines (métabolites moins volatiles et plus polaires) (6). Les principales réactions de dégradation sont les réactions d'oxydation, de réduction ou d'hydrolyse et les réactions de conjugaison avec acide glucuronique, sulfate, acétate ou acides aminés. En règle générale, le métabolisme résulte normalement en une détoxification de l'organisme mais parfois, une toxicité plus importante peut survenir. Par exemple, l'acétonitrile, le tétrachlorure de carbone, le chloroforme, le dichlorométhane, l'hexane, le méthanol, le trichloroéthylène et l'halothane conduisent à des métabolites plus toxiques. Le butane, les fluorocarbones, le tétrachloroéthylène et le 1,1,1-trichloroéthane sont majoritairement excrétés sous forme inchangé dans l'air expiré. Le tableau II rend compte du métabolisme de quelques substances volatiles $(1,6)$.

Tableau II : Métabolisme de quelques substances volatiles $(1,6,10,16,17,18)$.

\begin{tabular}{|l|l|}
\hline \multicolumn{1}{|c|}{ Substance } & \multicolumn{1}{c|}{ Métabolisme } \\
\hline Acétone & $\begin{array}{l}40 \text { à } 70 \% \text { inchangé } \\
\text { 2-propanol }\end{array}$ \\
\hline Acétonitrile & $\begin{array}{l}\text { Cyanures } \\
\text { Thiocyanate }\end{array}$ \\
\hline Benzène & $\begin{array}{l}12 \% \text { inchangé } \\
51 \text { à } 87 \% \text { de phénol, } 6 \% \text { de catéchol et } \\
\end{array}$ \\
& $2 \%$ d'hydroquinone excrété dans les urines \\
\hline
\end{tabular}

\begin{tabular}{|c|c|}
\hline Butane & $<1 \%$ de 2-butanol et de butanone \\
\hline Butanone & $\begin{array}{l}>99 \% \text { inchangé } \\
0,1 \% \text { de 3-hydroxybutanone excrété dans les urines }\end{array}$ \\
\hline $\begin{array}{l}\text { Tétrachlorure de } \\
\text { carbone }\end{array}$ & $\begin{array}{l}\text { Chloroforme } \\
\text { Dioxyde de carbone } \\
\text { Hexachloroéthane }\end{array}$ \\
\hline Chloroforme & $\begin{array}{l}20 \text { à } 70 \% \text { inchangé } \\
50 \% \text { de dioxyde de carbone } \\
\text { Diglutathionyldithiocarbonate }\end{array}$ \\
\hline Cyclohexanone & $\begin{array}{l}50 \% \text { de cyclohexanol excrété dans les urines } \\
\text { sous forme glucuronide }\end{array}$ \\
\hline Dichlorométhane & $50 \%$ de monoxyde de carbone \\
\hline Diméthylsulfoxide & $\begin{array}{l}3 \% \text { de diméthylsulfide dans l'air expiré } \\
18 \text { à } 20 \% \text { de diméthylsulfone excrété dans les urines }\end{array}$ \\
\hline Dioxane & Acide $\beta$-hydroxyéthoxyacétique excrété dans les urines \\
\hline Enflurane & $\begin{array}{l}>80 \% \text { inchangé et } 2,5 \% \text { d'acide } \\
\text { difluorométhoxydifluoroacétique }\end{array}$ \\
\hline Ether éthylique & $\begin{array}{l}1 \text { à } 2 \% \text { inchangé excrété dans les urines } \\
87 \% \text { inchangé dans l'air expiré } \\
1 \text { à } 5 \% \text { de dioxyde de carbone }\end{array}$ \\
\hline Acétate d'éthyle & $\begin{array}{l}\text { Ethanol } \\
\text { Acide acétique }\end{array}$ \\
\hline Ethylbenzène & $\begin{array}{l}5 \% \text { de mèthylphénylcarbinol excrété dans les urines } \\
\text { sous forme conjuguée } \\
64 \% \text { d'acide mandélic } \\
25 \% \text { d'acide phénylglyoxylique } \\
\end{array}$ \\
\hline Halothane & $\begin{array}{l}60 \text { à } 80 \% \text { inchangé } \\
<20 \% \text { de chlorotrifluoroéthane, de chlorodifluoroéthylène } \\
\text { et d'acide trifluoroacétique }\end{array}$ \\
\hline Hexane & $\begin{array}{l}\text { 2-hexanol excrété dans les urines sous formes glucuronide } \\
\text { 2-hexanone et 2,5-hexanedione }\end{array}$ \\
\hline Hydrocarbures & $\begin{array}{l}\text { Nombreux métabolites hydrosolubles } \\
\text { (alcools et phénols correspondants) }\end{array}$ \\
\hline Isobutane & $<1 \%$ de 2-méthyl-2-propanol \\
\hline Méthanol & $\begin{array}{l}>60 \% \text { de formaldéhyde } \\
\text { Acide formique excrété dans les urines }\end{array}$ \\
\hline Méthylbutylcétone & 2,5-hexanedione \\
\hline Méthyléthylcétone & $\begin{array}{l}\text { 2-butanol et butanediol excrétés dans les urines } \\
\text { sous forme glucuronide }\end{array}$ \\
\hline Méthyl-isobutylcétone & $\begin{array}{l}\text { 4-méthyl-2-pentanol et 4-hydroxy-4-méthyl-2-pentanone } \\
\text { excrétés dans les urines sous forme glucuronide }\end{array}$ \\
\hline Nitrite de butyle & 1-butanol \\
\hline Nitrite d'isobutyle & $\begin{array}{l}99 \% \text { de 2-méthyl-1-propanol } \\
\text { Nitrite inorganique }\end{array}$ \\
\hline Nitrite d'isopentyle & $\begin{array}{l}99 \text { \% de 3-méthyl-1-butanol } \\
\text { Nitrite inorganique }\end{array}$ \\
\hline Nitrite de pentyle & 1-pentanol \\
\hline Propane & \begin{tabular}{|l|} 
2-propanol \\
Acétone
\end{tabular} \\
\hline 2-propanol & 80 à $90 \%$ d'acétone \\
\hline Styrène & $\begin{array}{l}85 \% \text { d'acide mandélic excrété dans les urines } \\
10 \% \text { d'acide phénylglyoxylique } \\
\end{array}$ \\
\hline Tétrachloroéthylène & $\begin{array}{l}>90 \% \text { inchangé } \\
<3 \% \text { d'acide trichloroacétique excrété dans les urines }\end{array}$ \\
\hline Toluène & $\begin{array}{l}<20 \% \text { inchangé } \\
80 \% \text { d'acide benzoïque excrété dans les urines sous forme } \\
\text { conjugué avec la glycine (acide hippurique) } \\
1 \% \text { de } 0 \text {-,m- et } p \text {-crésol } \\
\end{array}$ \\
\hline 1, 1, 1-trichloroéthane & $\begin{array}{l}60 \text { à } 80 \% \text { inchangé } \\
2 \% \text { de } 2,2,2 \text {-trichloroéthanol excrété dans les urines } \\
0,5 \% \text { d'acide trichloroacétique }\end{array}$ \\
\hline Trichloroéthylène & $\begin{array}{l}16 \% \text { inchangé } \\
45 \% \text { de } 2,2,2 \text {-trichloroéthanol et } \\
32 \% \text { d'acide trichloroacétique excrétés dans les urines sous } \\
\text { forme glucuronide }\end{array}$ \\
\hline Xylènes & $\begin{array}{l}5 \% \text { inchangé } \\
2 \% \text { de xylénols } \\
95 \% \text { d'acide méthylbenzoïque excrété dans les urines } \\
\text { conjugué à la glycine (acide méthylhippurique) }\end{array}$ \\
\hline
\end{tabular}




\section{Solvants}

L'analyse par CPG est une alternative pour palier aux limitations des tests colorimétriques, tant en spécificité qu'en sensibilité. Les solvants, des plus légers (éthers) aux plus lourds (toluène, butanol), peut être séparés, identifiés et quantifiés. Les colonnes chromatographiques générallement utilisées sont de deux types : colonnes remplies (Carbowax) ou colonnes capillaire (phase de polyéthylène glycol). Pour les solvants, les détecteurs ECD, FID ou SM sont principalement utilisés. Un cas clinique rencontré à l'IML de Strasbourg concerne un homme de 28 ans, polytoxicomane connu, admis en service de réanimation. Un bilan urinaire par immunoanalyse est réalisé et s'avère positif pour les opiacés. La recherche à large spectre de substances volatiles par le couplage HS-CPG/SM a permis d'identifier et de doser du chloroforme dans le sang d'un laborantin suite à une intoxication par ce solvant organique (fig. 1). La concentration mesurée était de $6,7 \mathrm{mg} / \mathrm{L}$.

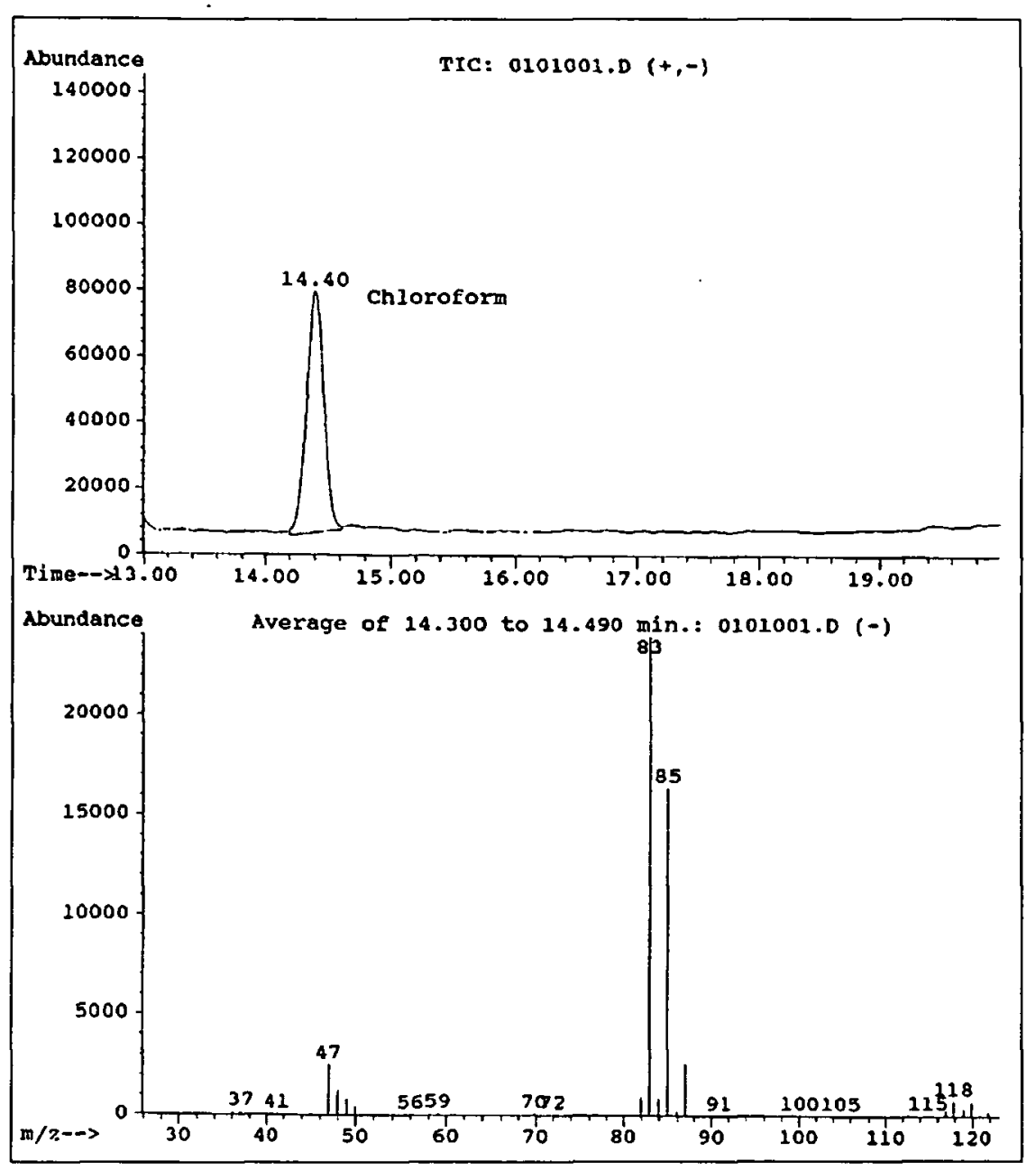

Figure 1 : Chromatogramme obtenu par HS-CPG/SM pour un prélèvement sanguin positif pour le chloroforme (temps de rétention 14,40 min, spectre $\mathrm{m} / \mathrm{z} 83,85$ et 47). Tracé obtenu après préparation "espace de tête", séparation chromatographique sur colonne capillaire HP-Wax et détection par spectrométrie de masse en mode "courant ionique total". La concentration mesurée était de $6,7 \mathrm{mg} / \mathrm{L}$.

\section{Le monoxyde de carbone ( $\mathrm{CO})$}

Le dosage du $\mathrm{CO}$ fait généralement appel à la dénaturation de la carboxyhémoglobine pour libérer le $\mathrm{CO}$ volatil. L'hydrolyse s'effectue générallement dans des conditions acides (acide sulfurique concentré) une fois l'échantillon déposés dans un tube hermétiquement clos. Cette étape peut être conduite à chaud pour accélerer le processus. Ce procédé peut être appliqué aux tissus dans les cas d'intoxication mortelle (16).

Une fois le $\mathrm{CO}$ volatilisé, un aliquot de vapeur est prélevé dans le flacon et injecté dans le chromatographe. La séparation est réalisée sur une colonne capillaire remplie d'un tamis moléculaire avec une taille des pores de $5 \mathrm{~A}^{\circ}$. Il s'agit de colonnes destinées à séparer aussi les constituants de l'air (azote, oxygène, dioxyde de carbone). La détection du CO se fait alors par un spectromètre de masse. Une alternative consiste en la réduction du $\mathrm{CO}$ en méthane pour une lecture sur un détecteur à ionisation de flamme (11).

Une affaire médicolégale publiée par Kintz et al. (16) rapporte le cas d'une femme retrouvée morte carbonisée. À l'autopsie, aucun prélèvement de sang ne peut être effectué, le corps ayant été exposés aux températures élevées de l'incendie. Dans ce cas, seuls des prélèvements tissulaires ont pu être réalisés. L'analyse de fragments de muscle a permis de déterminer la présence d'une teneur élevée $(18,5 \mu \mathrm{l} \mathrm{CO} / \mathrm{g})$ en monoxyde de carbone. La figure 2 a été obtenu par HS-CPG/SM à partir d'un échantillon de muscle prélevé sur la victime.

\section{Les cyanures $(\mathrm{CN})$}

La détermination du taux sanguin de cyanure est importante pour confirmer le diagnostic d'une intoxication par le cyanure. Leur dosage est réalisable par chromatographie gazeuse après acidification de l'échantillon et utilisation de chloramine $\mathrm{T}$ permettant de transformer $\mathrm{HCN}$ en $\mathrm{CNCl}$. La séparation chromatographique peut être réalisée sur colonne capillaire Poraplot $\mathrm{Q}$ et la détection par un détecteur à capture d'électrons, un détecteur azote-phosphore ou par un spectromètre de masse. Dans des cas d'intoxication aigüe par ingestion d'acétonitrile, le couplage HSCPG/SM a permis de déterminer des concentrations de cyanure sanguin de 2,4 et $4,5 \mathrm{ng} / \mathrm{mg}$ chez les deux victimes (17). Chez des victimes d'incendie, les concentrations enregistrées étaient entre 0,11 et $18,12 \mathrm{mg} / \mathrm{L}$ (18). La figure 3 illustre l'exemple d'un échantillon de sang prélevé sur une victime d'incendie et analysé par HS-CPG/SM avec détection en mode "ion sélectionné". La concentration de cyanure était de $234 \mathrm{ng} / \mathrm{ml}$. 


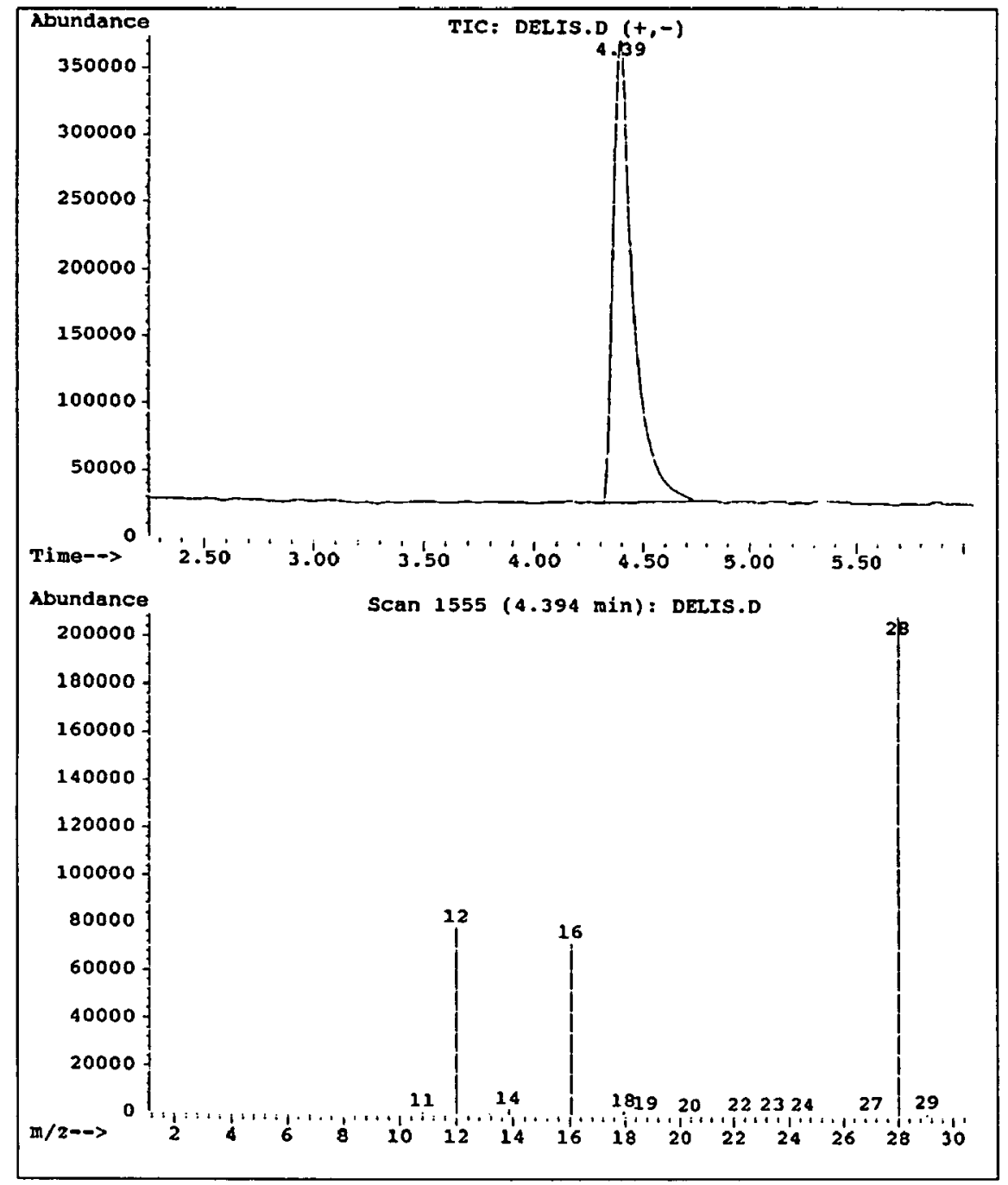

Figure 2 : Échantillon de muscle où la présence de monoxyde de carbone $(18,5 \mu \mathrm{l} \mathrm{CO} / \mathrm{g})$ a pu être détectée $(\mathrm{m} / \mathrm{z} 28,16$ et 12, temps de rétention 4,39 min). Tracé obtenu après préparation "espace de tête" séparation chromatographique sur molsive $5 A^{\circ}$ et détection par spectrométrie de masse en mode "ions sélectionnés".

\section{Les anesthésiques}

En pratique anesthésique, certains cas d'accident à l'halothane, un anesthésique volatile, ont été rapportés. Des études récentes ont essayé de préciser les conséquences d'une exposition du personnel de salles d'opération (1920). L'inhalation délibérée d'halothane ou même de chloroforme décrite dans le milieu médical et paramédical a parfois entraîné la mort de leurs utilisateurs. Le protoxyde d'azote est aussi détourné de son usage pour une utilisation récréative où sont recherchés ses effets euphorisants (21).

Dans le cas d'un double homicide suite à l'inhalation forcée de tissus imbibés d'halothane par les victimes, l'halothane a pu être identifié (22). Les concentrations mesurées par HS-CPG $(7,2 \mathrm{mg} / \mathrm{kg}$ chez la femme de 82 ans et $3,0 \mathrm{mg} / \mathrm{kg}$ chez l'homme de 75 ans) étaient inférieures aux concentrations mortelles citées dans la littérature (7 à $720 \mathrm{mg} / \mathrm{kg}$ ).

Récemment, le cas d'une infimière victime d'une intoxication par propofol a été rapportée. La recherche toxicologique dans le sang et les urines a démontré une exposition récente au Diprivan ${ }^{\circledast}$. La concentration de propofol déterminé par HS-CPG/SM dans le sang était

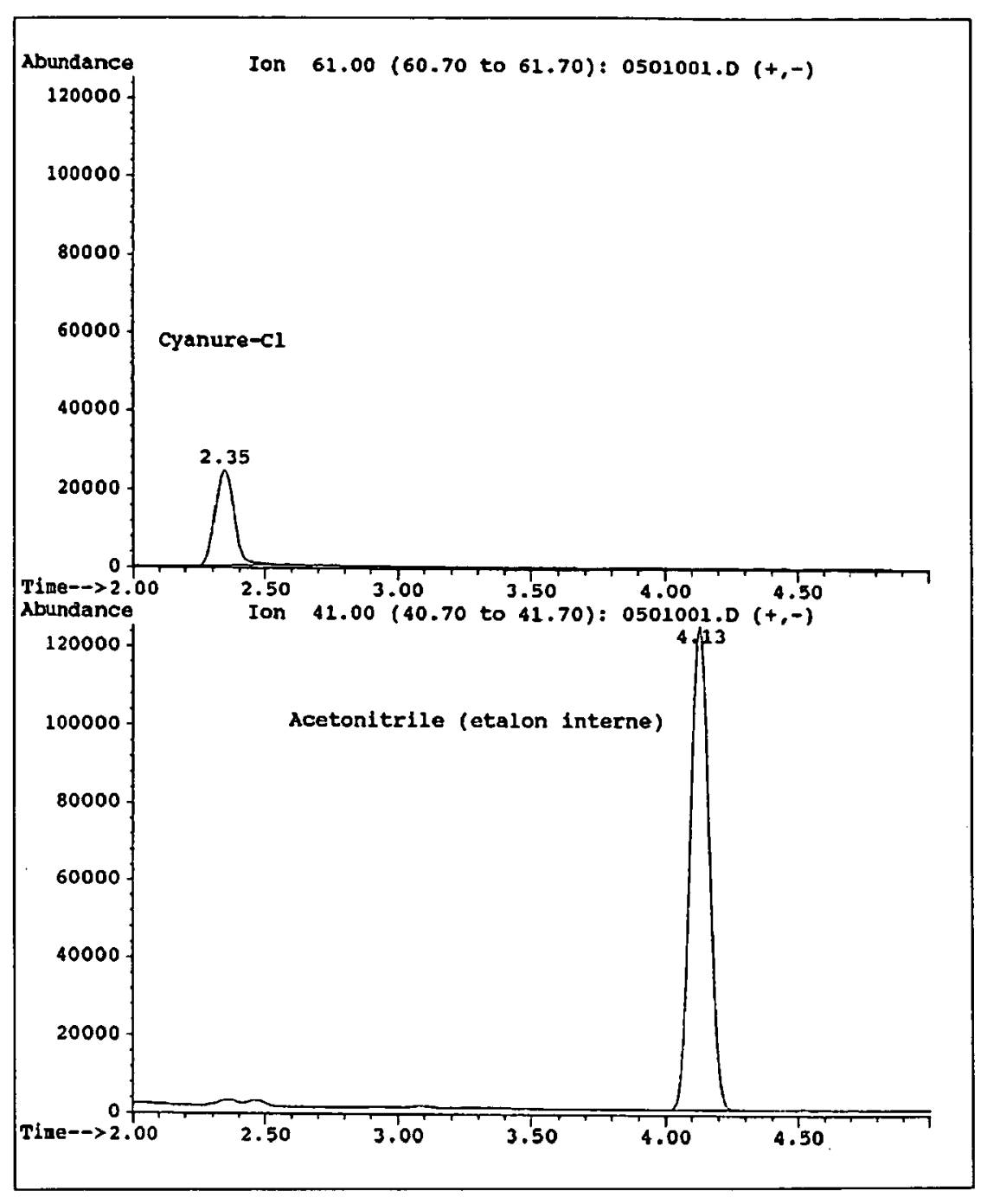

Figure 3 : Chromatogramme ionique obtenu par HSCPG/SM pour un échantillon de sang positif pour le cyanure (temps de rétention $2,35 \mathrm{~min}, \mathrm{~m} / \mathrm{z} 61,63,35$ ). La concentration calculée à l'aide de l'étalon interne était de 234 $n g / m l$.

de $39 \mathrm{ng} / \mathrm{ml}$ (23). La figure $4 \mathrm{a}$ été obtenue à partir de l'échantillon de sang.

\section{Un raticide : l' $\alpha$-chloralose}

Le test de coloration Fujiwara a l'avantage d'être rapide, mais il est peu spécifique car il ne permet pas d'identifier la substance en cause et ne donne qu'un résultat semi-quantitatif. La recherche d' $\alpha$-chloralose par le couplage HS/CPG/SM n'est possible qu'après hydrolyse de la liaison osidique entre l'hydrate de chloral et son sucre par un traitement acide (acide sulfurique concentré) à chaud $\left(3 \mathrm{~h}\right.$ à $\left.60^{\circ} \mathrm{C}\right)$. Le composé volatil (hydrate de chloral) est alors chromatographié sur colonne capillaire apolaire et détecté par spectrométrie de masse (24). La littérature internationale rapporte des concentrations létales variant de 13 à $410 \mathrm{mg} / \mathrm{L}(25)$.

\section{Les fréons}

Les fluorocarbones ont été introduits en 1940 en tant que réfrigérants et propulseurs d'aérosols grâce à leur propriété de composés inertes comparé au dioxyde de 
soufre, l'ammoniaque, le tétrachlorure de carbone et le chloroforme. Ils sont aussi utilisés en tant que solvants industriels dans le dégraissage et le séchage de métaux et plastiques afin de remplacer le trichloroéthylène et le tétrachloroéthylène.

$\mathrm{Au}$ départ, ils semblaient en général moins toxiques que les hydrocarbures chlorés, cependant, le FC113 a été décrit comme ayant un haut niveau de toxicité (26). Le trifluorométhane (FC11) est le plus connu pour ses effets dépresseurs. Les intoxications sévères font suite soit à une exposion accidentelle à une atmosphère riche en fréons soit à une prise intentionnelle dans un but récréatif. L'analyse des fréons dans les échantillons biologiques est généralement réalisée par HS-GC/FID $(15,27-29)$, par HS-GC/ECD $(15,30-31)$ ou par HSGC/MS (30-34). Deux autres techniques moins classiques ont été décrites : L'injection directe de sang en GC/MS (35) et la HS/MS sans séparation chromatographique (36).

En 1996, Kintz et al. (34) rapportèrent le cas de 2 décès liés à une exposition accidentelle au FC22 lors de la vérification d'un système de réfrigération dans la cale d'un bateau. Les analyses par HS-GC/MS confirmèrent

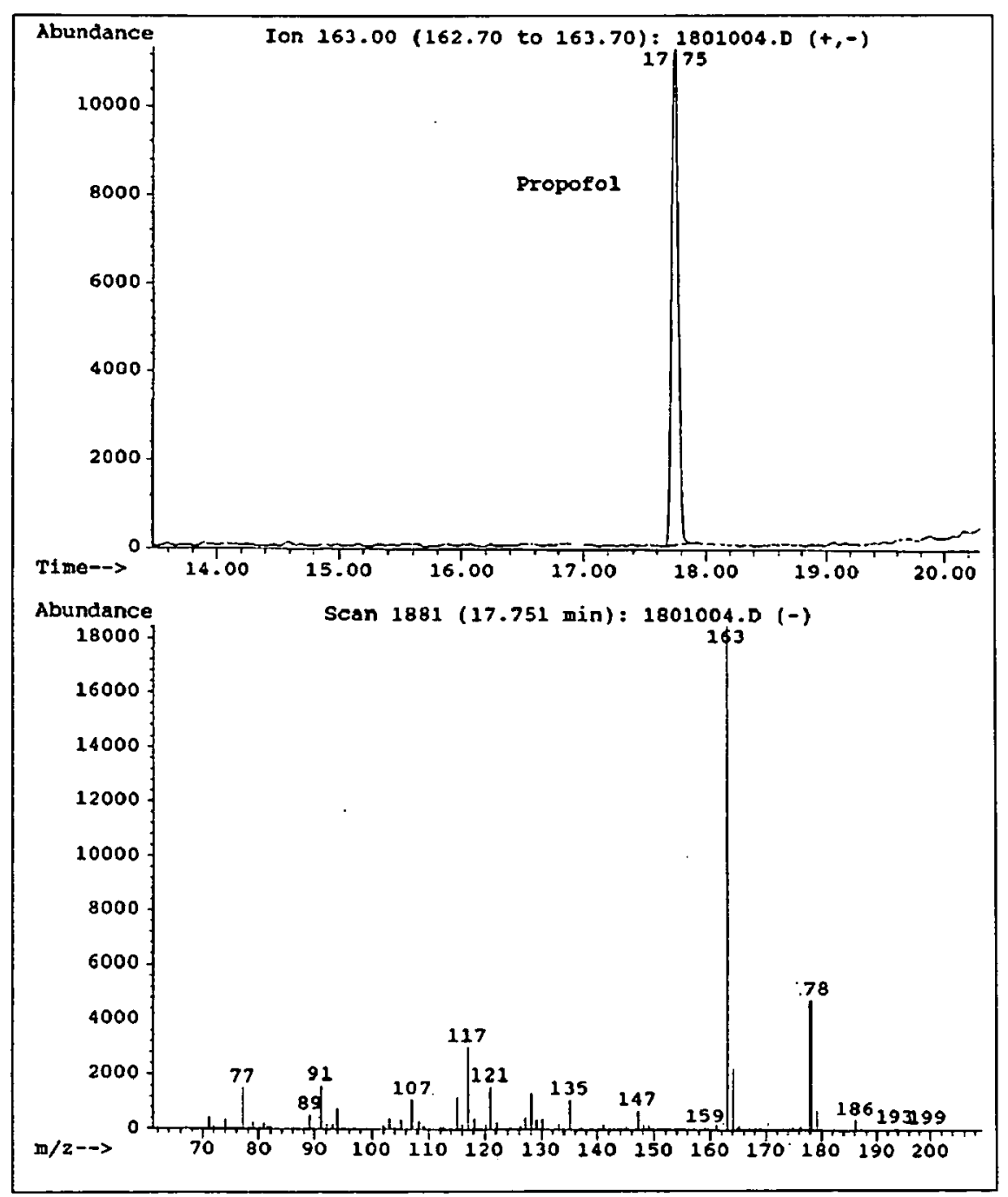

Figure 4 : Échantillon sanguin positif pour le propofol révèlé par HS-CPG/SM (temps de rétention 17,75 $\mathrm{min}, \mathrm{m} / \mathrm{z}$ 163, 178, 117). Tracé obtenu après préparation "espace de tête", séparation chromatographique sur colonne capillaire HP-Wax et détection par spectrométrie de masse en mode "courant ionique total". la présence de FC22 aux concentrations de 37 et 26 $\mu \mathrm{l} / \mathrm{ml}$ dans le sang des deux victimes. Le FC22 a une faible affinité pour le sang et donc sa concentration plasmatique est directement proportionnelle au degré d'exposition (37). Lors d'études contrôlées chez l'homme, il fût montré que l'excrétion urinaire du FC22 est très faible (37-38). La figure 5 représente un tracé obtenus après préparation "espace de tête", séparation chromatographique sur colonne apolaire (HP-5MS), et détection par spectrométrie de masse de deux fluorocarbones, le FC13B1 ou bromotrifluoromethane et le FC 12 ou dichlorodifluorométhane utilisé ici comme étalon interne. La concentration sanguine de FC13B1 $(\mathrm{m} / z 6979129$ 148) était dans ce cas de $17 \mu \mathrm{l} / \mathrm{ml}$.

\section{Les gaz lacrymogènes}

Les gaz lacrymogènes contenus dans les bombes de défense, couramment utilisés pour neutraliser un sujet, sont réputés dangereux. Un exemple de produit commercialisé sous la dénomination "Bombe anti-agressive à 20 $\%$, à usage militaire, police et gendarme" contient 51 gr de substance active, l'ortho-chlorobenzylidène malononitrile (CS), à $20 \%$ dans un solvant et 36 gr de propulseur.

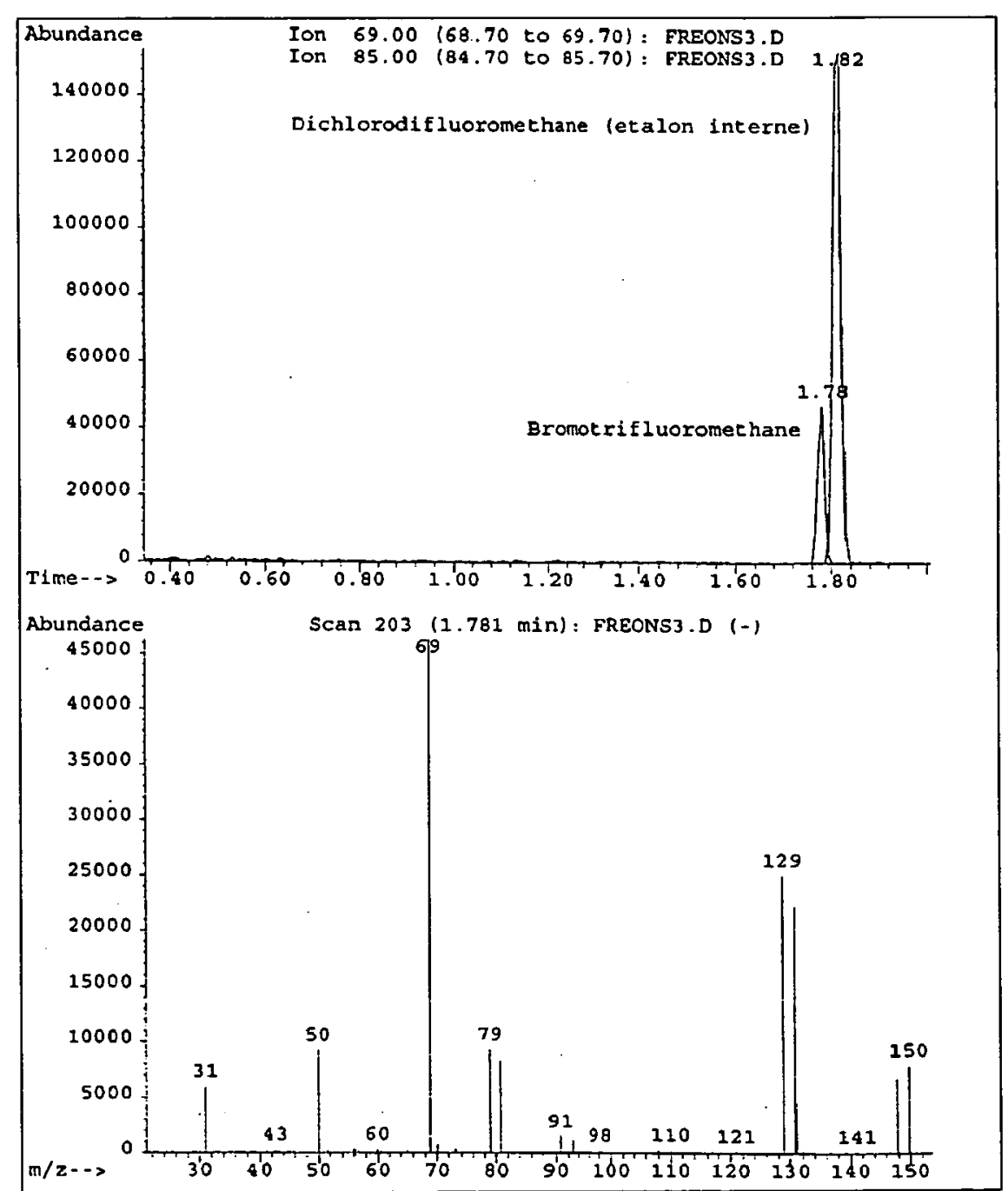

Figure 5 : Tracé obtenu par HS-CPG/SM pour un échantillon sanguin positif en bromotrifluorométhane $(17 \mathrm{\mu l} / \mathrm{ml})$. La séparation chromatographique a été réalisée sur colonne apolaire HP-5MS. Le dichlorodifluorométhane $(\mathrm{m} / z) 85$, 101 et 50) a été utilisé pour la quantification. 
La substance active peut aussi être:

- de l' $\alpha$-chloroacétophénone ( $\mathrm{CN})$

- du dibenz(b,f)-1,4-oxazépine (CR)

Les méthodes analytiques publiées pour la détection de ces composés lacrymogènes sont la spectrophotométrie ultraviolette ou infrarouge (39), la GC ou la GC/MS (40-41) et IMS (ion mobility spectrometry) (42).

\section{Les nitrites d'alkyle ou aliphatiques}

Les nitrites d'alkyle sont des esters aliphatiques d'acides nitreux avec une structure générale $\mathrm{R}-\mathrm{O}-\mathrm{N}=\mathrm{O}$.

Le nitrite d'amyle a été introduit en médecine en 1867 par Lauder Brunton dans le traitement des angines de poitrine. Dans les années 1950, les médecins américains ont constaté une utilisation non médicale du nitrite d'amyle comme aphrodisiaque. C'est à ce moment là qu'apparaît leur surnom de "poppers". A la fin des années 1960 , le nitrite d'amyle fut considéré comme un produit pharmaceutique nécessitant une prescription médicale. Il a donc été remplacé par le nitrite de butyle ou d'isobutyle et dernièrement par le nitrite de propyle.

Les nitrites volatils, et plus particulièrement le nitrite d'isobutyle, font partis des aphrodisiaques les plus utilisés à l'heure actuelle.

Plusieurs cas d'intoxication par ingestion de ces composés ont été rapportés (43-45). Deux autres cas de déces suite à l'inhalation de nitrite de butyle ont été rapportés par Shesser (46) et Bradberry (47). Dans les cas d'intoxication aiguë aux nitrites d'alkyle, la methémoglobinémie et/ou l'anoxie sont les principales causes de décès. Leur dosage peut être réalisé par spectrophotométrie ultraviolette, par infrarouge, par HPLC et par GC/MS (48).

\section{L'hydrogène sulfuré}

L'hydrogène sulfuré est l'un des gaz courants les plus toxiques, incolore et dont l'odeur caractéristique ("oeuf pourri") permet la détection à faible concentration. Les sources naturelles d'hydrogène sulfuré sont les gaz volcaniques et souterrains (caves, mines, etc.) (49), les gisements de pétrole (50) et de gaz naturel (51) ; c'est aussi un produit de la décomposition bactérienne anaérobie des matières organiques (égouts, fosses septiques, fosses à purin, etc.) (52-56). Enfin, un grand nombre de processus industriels mettent en jeu l'hydrogène sulfuré, soit comme matière première (57), soit comme produit secondaire ou déchet de fabrication (52, 58-60, 60-61). Le dosage des ions sulfures est généralement réalisé par ionométrie (60). Dans les rares intoxications létales décrites, le taux sanguin de sulfures variait entre 1,7 et $14,2 \mathrm{mg} / \mathrm{l}(55,60,62)$. A 500 ppm, il entraîne une brusque perte de connaissance $(55,63-64)$. En intoxication aiguë $(1000 \mathrm{ppm})$, il entraîne la mort en quelques secondes.

Le couplage HS/CPG/SM permet de mettre en évidence directement l'hydrogène sulfuré après hydrolyse acide (65).

\section{Conclusion}

Dans le cadre d'une intoxication par substance volatile, la préparation "espace de tête" suivie d'un couplage chromatographie en phase gazeuse couplée à un détecteur de masse représente la méthode de choix pour l'identification et le dosage du toxique impliqué dans les diverses matrices biologiques. Dans bien des cas, cette technique représente un complément idéal des analyses toxicologiques classiques.

\section{Références}

1. Haguenoer J.M., Furon D. Le comportement en milieu de travail ; problème d'éducation pour la santé. Toxicologie et Hygiène industrielles. Paris : Techniques et documentation $1983 ; 10: 91-198$.

2. Jouglard J., Jean Ph., David J-M., Arditti J., Aicardi F., Le sniffing : jeu d'écolier ou toxicomanie? Résultat d'une enquête concernant 49 écoliers sniffeurs. Méd. et Hyg. $1984 ; 42: 2479-87$.

3. Sentilhes N., Garnier R., Inhalation de solvants chez les enfants d'âge scolaire. Le Concours Médical 1982 ; 104 : 4337-41.

4. Berthault F., Tracqui A., Kintz P., Mangin P., Intoxication au trichloréthylène et conservation des prélèvements. Toxicorama $1995 ; 7: 27-32$.
5. Gill R., Hatchett S.E., Osselton M.D., Wilson H.K., Ramsey J.D., Sample handling and storage for the quantitative analysis of volatile compounds in blood: the determination of toluene by headspace gas chromatography. J. Anal. Toxicol. $1988 ; 12: 141-6$.

6. Flanagan R.J., Streete P.J., Ramsey J.D. Volatile substance abuse: Practical guidelines for analytical investigation of suspected cases and interpretation of results. Vienna : United Nations International Drug Control Programme $1997: 2-38$.

7. Catilina P., Chamoux A., Etude de l'élimination urinaire de l'acide hippurique chez des travailleurs exposés à de faibles concentrations atmosphériques de toluène. Arch. Mal. Prof. $1980 ; 41$ : 141-6. 
8. Tardif R., Brodeur J., Plaa G.L., Simultaneous High-performance liquid chromatographic analysis of hippuric acid and ortho-, meta- and para-methylhippuric acids in urine. J. Anal. Toxicol. 1989 ; 13 : 313-6.

9. Wilczok T., Bieniek G., Urinary hippuric acid concentration after occupational exposure to toluene. Br. J. Industrial. Med. 1978 ; 35 : 330-4.

10. Clarke's isolation and identification of drugs in pharmaceuticals, body fluids, and post-mortem material. London : The Pharmaceutical Press. 1986 : 153.

11. Vallon J.J. Intoxication oxycarbonée. Dans : Kintz P. ed. Toxicologie et pharmacologie médicolégales. Amsterdarm : Elsevier, 1998 : 127-45.

12. Theodoridis G., Koster E.H.M., de Jong G.J., Solid-phase microextraction for the analysis of biological samples. J. Chromatogr. B $2000 ; 745: 49-82$.

13. Brewer W.E., Galipo R.C., Morgan S.L., Habben K.H., The confirmation of volatiles by solid-phase microextraction and GC/MS in the investigation of two traffic fatalities. J. Anal. Toxicol. $1997 ; 21$ : 286-90.

14. Dehon B., Humbert L., Devisme L., Stievenart M. Mathieu D., Houdret N., Lhermitte M., Tetrachloroethylene and trichloroethylene fatality: Case report and simple headspace SPME-capillary gas chromatographic determination in tissues. J. Anal. Toxicol. $2000 ; 24: 22-6$.

15. Ramsey J.D., Flanagan R.J., Detection and identification of volatile organic compounds in blood by headspace gas chromatography as an aid to the diagnosis of solvent abuse. J. Chromatogr. 1977 ; 240 : 423-44.

16. Kintz P., Cirimele V., Marquet P., Deveaux M., Ludes B., Dosage du monoxyde de carbone par HS-GC-MS. J. Med. Leg. Droit Med. 2000 ; 43 : 145-8.

17. Jones A.W., Lâofgren A., Eklund A., Grundin R., Two fatalities ingestion of acetonitrile : limited specificity of analysis by headspace gas chromatography. J. Anal. Toxicol. $1992 ; 16$ : 104-6.

18. Shiono H., Maseda C., Akane A., Matsubara K., Rapid and sensitive quantitation of cyanide in blood ans its application to fire victims. Am. J. Forensic Pathol. 1991; $12: 50-3$

19. De Zotti R., Negro C., Gobbato F., Results of hepatic and hemopoietic controls in hospital personnel exposed to waste anesthetic gases. Int. Arch. Occup. Environ. Health $1983 ; 52: 33-41$.

20. Stoklow M., Troviller P., Stieglitz P., L'exposition aux gaz anesthésiques : risques et prévention. Sem. Hop. Paris 1983 ; 59 : 2081-7.

21. Boutron C., Mathieu-Nolf M., Pety N., Deveaux M., Utilisations détournées du protoxyde d'azote. ATA 2000 ; 12 : 208-13.

22. Musshoff F., Junker H., Madea B., Rapid analysis of halothane in biological samples using headspace solidphase microextraction and gas chromatography-mass spectrometry- A case of a double homicide. J. Anal. Toxicol. $2000 ; 24: 372-6$.

23. Cirimele V., Kintz P., Doray S., Ludes B., Determination of chronic abuse of the anesthetic agents midazolam and propofol by hair analysis. Int. J. Leg. Med. soumis
24. Kintz P., Doray S., Cirimele V., Ludes B., Testing for alpha-chloralose by headspace-GC-MS. A case report. Forensic Sci. Int. 1999 ; 104 : 59-63.

25. Schmid M., Iten P., A fatal poisoning involving alphachloralose (abstract). In : Spieler V. ed. Proceedings of the 1994 Joint TIAFT-SOFT International Meeting, Tampa, USA, October 31-November 4, 1994, pp140-1.

26. Aviado D.M., Toxicity of aerosols. J. Clin. Pharmacol. $1975 ; 15: 86-104$.

27. Jefferson I.G., Accidental death of child playing with deodorant aerosol. Lancet $1978 ; 1$ : 779.

28. Morita M., Miki A., Kazama H., Sakata M., Case report of deaths caused by Freon gas. Forensic Sci. Int. 1977 ; $10: 253-60$.

29. Standefer J.C., Death associated with fluorocarbon inhalation : Report of a case. J. Forensic Sci. 1975 ; 20 : 54851 .

30. McGee M.B., Meyer R.F., Jejurikar S.G., A death resulting from trichlorotrifluoroethane poisoning. J. Forensic Sci. $1990 ; 35: 1453-60$.

31. Poklis A., Determination of fluorocarbon 11 and fluorocarbon 12 in post-mortem tissues : a case report. Forensic Sci. Int. $1975 ; 5: 53-9$.

32. Backer R.C., Pisano R.V., Gas chromatography-mass spectrometry of fluorocarbons 11 and 12 in biologic specimens. Clin. Toxicol. $1978 ; 12: 69-75$.

33. Hamill J., Kee T.G., The detection of aerosol propellants in body fluids and tissue by gas chromatography-mass spectrometry. J. Forensic Sci. Soc. 1991 ; 31 : 301-7.

34. Kintz P., Baccino E., Tracqui A., Mangin P., Headspace GC/MS testing for chlorodifluoromethane in two fatal cases. Forensic Sci. Int. 1996 ; 82 : 171-5.

35. Dollery C.T., Davies D.S., Draffan G.H., Williams F.M., Connolly M.E., Blood concentrations in man of fluorinated hydrocarbons after inhalation of pressurized aerosols. Lancet $1970 ; 2$ : 1164-6.

36. Urich R.W., Bowerman D.L., Wittenberg P.H., Pierce A.F., Schisler D.K., Levisky J.A., Pflug J.L., Headspace mass spectrometric analysis for volatiles in biological specimens. J. Anal. Toxicol. 1977 ; 1 : 195-9.

37. Wollen B.H., Marsh J.R., Mahler J.D., Auton T.R., Makepeace D., Cocker J., Blain P.G., Human inhalation pharmacokinetics of chlorodifluoromethane (HCFC 22). Int. Arch. Occup. Environ. Health 1992 ; 64 : 383-7.

38. Groppi A., Polettini A., Lunetta P., Achille G., Montagna M., A fatal case of trichlorofluoromethane (Freon 11) poisoning. Tissue distribution study by gas chromatography-mass spectrometry. J. Forensic Sci. 1994 ; 39 : 8716.

39. Sreenivasen V.R., Boese R.A., Identification of lachrymators. J. Forensic Sci. $1970 ; 15: 433-42$.

40. Ferslew K.E., Orcutt R.H., Hagardorn A.N., Spectral differentiation and gas chromatographic/mass spectrometric analysis of the lacrimators 2-chloroacetophenone and ochlorobenzylidene malononitrile. J. Forensic Sci. 1986 ; $31: 658-65$.

41. Nowicki J., Analysis of chemical protection sprays by gas chromatography/mass spectrometry. J. Forensic Sci. $1982 ; 27$ : 704-9. 
42. Allison G., McLeod C.W., Characterization of lachrymators by ambient temperature ion mobility spectrometry. $\mathrm{J}$. Forensic Sci. 1997 ; 42 : 312-5.

43. Cohen S. The volatile nitrites. J. A. M. A. $1979 ; 241$ : 2077-8.

44. Dixon D.S., Reisch R.F., Santinga P.H., Fatal methemoglobinemia resulting from ingestion of isobutyl nitrite, a "room odorizer" widely used for recreational purposes. J. Forensic Sci. 1981 ; 26 : 587-93.

45. O'Toole J.B., Robbins G.B., Dixon D.S., Ingestion of isobutyl nitrite, a recreational chemical of abuse, causing fatal methemoglobinemia. J. Forensic Sci. 1987 ; 32 : 1811-14.

46. Shesser R., Mitchell J., Edelstein S., Methemoglobinemia from isobutyl nitrite preparations. Ann. Emerg. Med. $1981 ; 10: 262-4$.

47. Bradberry S.M., Whittington R.M., Parry D.A., Vale J.A., Fatal methemoglobinemia due to inhalation of isobutyl nitrite. Clin. Toxicol. 1994 ; 32 : 179-84.

48. Schmitt G., Bogusz M., Poppers (isobutilnitrit)-"Das Cocain der armen Leute". Beitr. Ger. Med. 1989 ; 47 : 63 8.

49. Milby T.H., Hydrogen sulfide intoxication. J. Occup. Med. $1962 ; 4: 431-7$.

50. Goodwin L.R., Francom D., Dieken F.P., Taylor J.D., Warenycia M.W., Reiffenstein R.J., Dowling G., Determination of sulfide in brain tissue by gas dialysis/ion chromatography: postmortem studies and two case reports. J. Anal. Toxicol. $1989 ; 13: 105-9$.

51. Wasch H.H., Estrin W.J., Yip P., Bowler R., Cone J.E., Prolongation of the P-300 latency associated with hydrogen sulfide exposure. Arch. Neurol. 1989 ; 46 : 902-4.

52. Guillon F., Mignee C., Girard Wallon C., Durigon M., A propos de cinq intoxications aiguës mortelles mettant en cause l'hydrogène sulfuré. Arch. Mal. Prof. 1983 ; 44 : 483-8.

53. Landrigan P.J., Miller B. The Arjenyattah epidemic : home interview data and toxicological aspects. Lancet 1983 ; ii : $1474-6$.
54. Morse D.L., Woodburg M.A., Rentmeester K., Farmer D., Death caused by fermenting manure. J. A. M. A. $1981 ; 245: 63-4$.

55. Osbern L.N., Crapo R.O., Dung lung : a report of toxic exposure to liquid manure. Ann. Inter. med. 1981 ; 95 : 312-4.

56. Penneau D., Chennebault J.M., Petiet G., Domont A., Loriot J., Proteau J., Intoxication par l'hydrogène sulfuré en milieu rural. Arch. Mal. Prof. 1982 ; 43 : 133-5.

57. Miller N., Gold M., Organic solvent and aerosol abuse. Am. Fam. Phys. $1991 ; 44$ : 182-99.

58. Aaserud O., Hommeren O.J., Tvedt B., Nakstad P., Mowe G., Efskind J., Russell D., Jorgensen E.B., Nyberg-Hansen R., Rootwelt K., Carbon disulfide exposure and neurotoxic sequelae among viscose rayon workers. Am. J. Ind. Med. 1990 ; 18 : 25-37.

59. Beasley R.W.R., The eye and hydrogen sulphide. Br. J. Industr. Med. 1963 ; 20 : 32-4.

60. Mc Analley B.H., Lowry W.T., Oliver R.D., Garriott J.C., Determination of inorganic sulfide and cyanide in blood using specific ion electrodes : application to the investigation of hydrogen sulfide and cyanide poisoning. $\mathbf{J}$. Anal. Toxicol. $1979 ; 3: 111-4$.

61. Stine R.J., Slosberg B., Beacham B.E., Hydrogen sulfide intoxication : a case report and discussion of treatment. Ann. Intern. Med. 1976 ; 85 : 756-8.

62. Tracqui A., Kintz P., Pagel E., Mangin P., Intoxication mortelle par l'hydrogène sulfuré. J. Méd. Strasbourg $1991 ; 22: 172-5$.

63. Burnett W.W., King E.G., Grace M., Hall W.F., Hydrogen sulfide poisoning : review of 5 years experience. Can. Med. Assoc. J. 1977 ; 117 : 1277-81.

64. Raymond V., Cavigneaux A., Bernuchon J., Hervy J., Intoxications accidentelles dans une cuve de tannerie. Arch. Mal. Prof. 1963 ; 24 : 307-10.

65. Kintz P., Cirimele V., Ludes B., Direct quantitation of hydrogen sulfure by HS/GC/MS in fatal cases. Forensic Sci. Int. soumis. 